\title{
Alcoolisation d'un hépatocarcinome guidée par échoendoscopie
}

\author{
P. GAST, J. DELWAIDE, V. GILLARD, J. BELAÏCHE \\ CHU Liège Sart Tilman, Service de gastroentérologie, Liège (Belgique)
}

\begin{abstract}
L'échoendoscopie est un examen qui occupe une place importante dans les bilans oncologiques. Depuis quelques années, elle s'avère aussi être une aide précieuse pour certaines thérapeutiques.
\end{abstract}

Nous relatons ici, la réalisation d'une alcoolisation guidée par échoendoscopie d'un hépatocarcinome du segment 1 . La décision de réaliser une alcoolisation avait été prise en consultation pluridisciplinaire d'oncologie, chez un patient non éligible pour la chirurgie du fait de son âge (79 ans) et de la localisation tumorale. Le choix d'un guidage échoendoscopique avait été fait en concertation avec les experts radiologues, compte tenu de la morphologie du patient. La préparation et l'installation du malade étaient conformes à celles d'une FNA classique. Une aiguille de $22 \mathrm{G}$ a été utilisée et une première séance d'alcoolisation finalisée. Aucune complication immédiate n'a été notée et le patient a pu quitter le service le jour même. Seuls des accès fébriles, généralement considérés comme la traduction clinique de la nécrose tumorale, ont été rapportés, aisément contrôlés par la prise de paracétamol. Un CT scan de contrôle réalisé un mois plus tard, a démontré des modifications significatives de l'aspect tumoral, mais a aussi malheureusement dévoilé d'autres lésions passées inaperçues lors du premier bilan. Tout espoir d'être curatif étant alors perdu, les autres séances ont été annulées et la situation expliquée au patient qui depuis, est décédé des suites de l'évolution de sa maladie.

\section{Utilisation pratique de l'écho-endoscopie (EUS) pour le suivi quotidien des patients cirrhotiques}

\author{
P. CASTELLANI, C. BOUSTIÈRE, O. LE BARS, M. BOURLIÈRE \\ Hôpital Saint-Joseph, Marseille (France)
}

L'EUS est utilisée quotidiennement dans la prise en charge de pathologies biliaires ou tumorales. Par contre, son utilisation en matière d'hypertension portale (HTP) n'est pas répandue et probablement sous évaluée. Les situations cliniques pour la prise en charge des patients cirrhotiques sont nombreuses et leur exploration passe par l'échographie, l'endoscopie diagnostique et thérapeutique, le scanner ou l'IRM. Les études sont cependant nombreuses pour valider l'utilité de l'EUS dans ces différentes situations, à tous les stades de la maladie cirrhotique. Dans le diagnostic précoce de l'hypertension portale, dans le bilan morphologique du système porte, dans l'approche hémodynamique de l'HTP, avant traitement médical, dans le suivi du traitement ou dans les modifications de ce traitement, dans les indications de dérivations porto systémiques radiologiques ou chirurgicales, dans le suivi de ces dérivations, pour la vision directe des circulations collatérales et leur rôle dans l'hémorragie. L'EUS permet également de réaliser des biopsies de petites lésions hépatiques difficilement accessibles par d'autres techniques, de biopsier des thrombus cruoriques ou tumoraux de la veine porte, de guider des injections dans des collatérales hémodynamiquement efficaces. Le doppler, finalement peu utilisé dans l'EUS biliaire ou tumorale, trouve, dans l'HTP, toute sa place avec des explorations simples et des index hémodynamiques plus complexes, dont certains sont encore à valider. Dans notre pratique quotidienne, l'EUS est donc largement utilisée pour la prise en charge des patients cirrhotiques, du début de leur maladie, lors des complications (ascite ou hémorragie) ou au stade de la transplantation. L'EUS, dans notre expérience, modifie directement le suivi et les indications thérapeutiques de ces patients. Des études prospectives, utilisant l'EUS, sont également en cours pour avoir une approche hémodynamique de la cirrhose et de ses complications. 